\title{
Opinionated article
}

\author{
Stephen R. Forrest*
}

\section{Waiting for Act 2: what lies beyond organic light- emitting diode (OLED) displays for organic electronics?}

https://doi.org/10.1515/nanoph-2020-0322

Received June 9, 2020; accepted July 23, 2020; published online August 24, 2020

\begin{abstract}
Organic light-emitting diode (OLED) displays are now poised to be the dominant mobile display technology and are at the heart of the most attractive televisions and electronic tablets on the market today. But this begs the question: what is the next big opportunity that will be addressed by organic electronics? We attempt to answer this question based on the unique attributes of organic electronic devices: their efficient optical absorption and emission properties, their ability to be deposited on ultrathin foldable, moldable and bendable substrates, the diversity of function due to the limitless palette of organic materials and the low environmental impact of the materials and their means of fabrication. With these unique qualities, organic electronics presents opportunities that range from lighting to solar cells to medical sensing. In this paper, we consider the transformative changes to electronic and photonic technologies that might yet be realized using these unconventional, soft semiconductor thin films.
\end{abstract}

Keywords: lighting; organic semiconductor; reliability; solar cell; thin film transistor.

\section{An introduction to organic electronics}

The field of organic electronics, now in its 70th year since the identification of semiconducting properties of violanthrone by Akamatsu and Inokuchi [1], has enjoyed an extended period of discovery of the characteristics of disordered organic materials, ultimately leading to the

*Corresponding author: Stephen R. Forrest, Departments of Electrical Engineering and Computer Science, Physics, and Materials Science and Engineering, University of Michigan, Ann Arbor, MI 48104, USA, E-mail: stevefor@umich.edu astonishing success of organic light-emitting diodes (OLEDs). This technology platform has launched a revolution in information displays and lighting, while motivating researchers worldwide to explore a vast variety of new materials with intriguing optical and electronic properties that were never imagined in those early days of discovery [2]. The fundamental discoveries encouraged the small community of researchers to consider if there were any practical outcomes that could be achieved using organic semiconductors. Some of the first devices to exploit these "soft" materials were memories and solar cells. But compared to conventional semiconductor devices (most notably $\mathrm{Si}$ ), the performance of organic devices was depressingly inferior, and worse, they did not last very long. It was their lack of stability that has given rise to a myth that persists up to the present day: organic devices are inherently unstable. We will return to this issue below.

Formally, an organic material is one that contains a carbon-hydrogen bond. By this definition, fullerenes (e.g., $\mathrm{C}_{60}$ ), carbon nanotubes and graphene are not organic compounds. But, more practically, it can be considered to be one of a class of carbon-rich compounds. In the context of this paper, the organic materials of interest in electronics and photonics are semiconductors whose energy gaps are typically between 0.75 and $3.5 \mathrm{eV}$.

The pace of these first, tentative steps in exploiting the unlimited variety of organic materials for optoelectronic applications took an immense leap by the publication of two papers out of Eastman Kodak in 1986 and 1987. The first one, by C. W. Tang, announced the demonstration of an organic solar cell with $1 \%$ solar to electrical power conversion efficiency (PCE). The efficiency was not particularly high - that is not what made this demonstration so notable [3]. What was different is that this was a device that mimicked an inorganic $\mathrm{p}-\mathrm{n}$ junction by combining two different organic semiconductor layers, one an electron donor (D) and the other an acceptor (A), into a bilayer cell. For the first time, the current-voltage characteristics showed nearly ideal rectifying characteristics that up to that time were only found in inorganic junction 


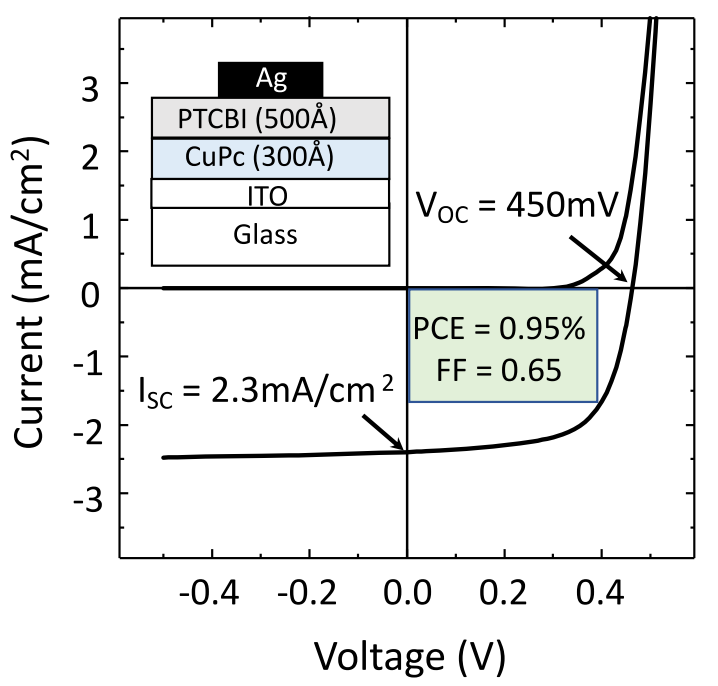

Figure 1: Current-voltage characteristics of the bilayer organic photovoltaic cell shown schematically in the inset [3]. The chemicals used are the acceptor, 3,4,9,10-perylenetetracarboxylic bisbenzimidazole (PTCBI), and the donor, copper phthalocyanine (CUPC). Indium tin oxide (ITO) serves as the transparent anode and $\mathrm{Ag}$ as the cathode. The short circuit current $\left(I_{S C}\right)$, open circuit voltage $\left(V_{O C}\right)$, power conversion efficiency $(P C E)$ and fill factor $(F F)$ under AM2 simulated illumination at $75 \mathrm{~mW} / \mathrm{cm}^{2}$ intensity are indicated. Here, the maximum power generated by the cell is equal to the area in the shaded rectangle and is given by $P C E_{\max }=F F \cdot I_{S C} \cdot V_{O C} / P_{\text {sun }}$, where $P_{\text {sun }}$ is the incident solar power intensity.

diodes (see Figure 1). From that demonstration forward, all organic solar cells have used the same basic D-A heterojunction (HJ) concept.

The second paper in 1987 was in many ways similar to the solar cell. Again, C. W. Tang, this time in collaboration with Steven van Slyke announced the successful demonstration of a bilayer organic light-emitting diode (OLED) [4]. The OLED, like the solar cell, had a clear rectifying behavior. But most interestingly, it exhibited bright green emission due to exciton recombination on one of the molecules forming the bilayer, namely 8-hydroxyquinoline Aluminum $\left(\mathrm{Alq}_{3}\right)$ with an external quantum efficiency of $1 \%$. This was inferior compared to inorganic semiconductors at that time based on GaAs or InP, but the very thin films ( 100 nm) comprising the device were grown on a glass substrate. Figure 2 shows a simplified, generic structure of modern OLEDs. Perhaps, if organics could only last long enough, they would be the foundation of a new generation of displays that, at that time, was dominated by cathode ray tubes and the emerging liquid crystal displays (LCDs).

While OLEDs looked promising (their colors could easily be modified across the visible spectrum by implementing only minor modifications to the chemical structures of the fluorescent emitting molecules or fluorophores), their lifetimes and efficiencies still fell short of what was already being

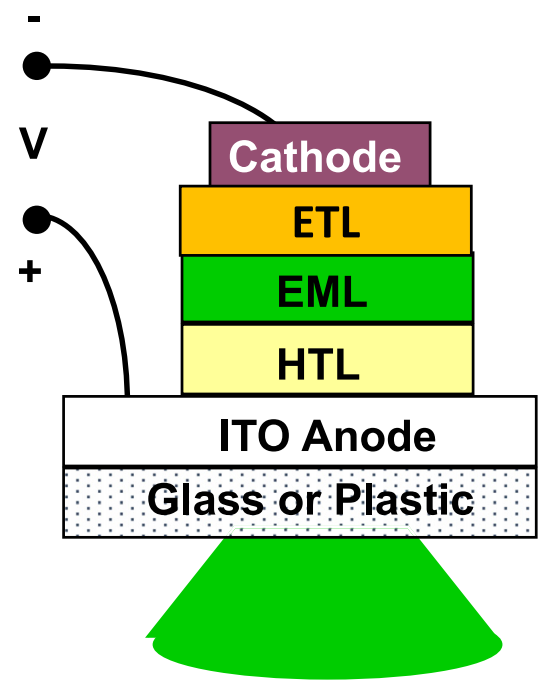

Figure 2: A simplified organic light-emitting diode (OLED) structure indicating the contacts, electron transport layer (ETL), light emission layer (EML) and hole transport layer (HTL). The EML typically comprises a conductive organic host doped at low density with an emissive fluorescent or phosphorescent emitting molecule. The entire device thickness is $\sim 100 \mathrm{~nm}$.

achieved by LCDs. This situation changed dramatically with the introduction of electrophosphorescence by Baldo et al. [5, 6] that almost immediately led to OLEDs with $100 \%$ internal quantum efficiency [7]. Briefly, molecular excited states, or excitons, fall into two categories based on the spin of the excited electron: singlets and triplets. Singlets have odd symmetry under spin exchange, leading to rapid, fluorescent emission by transitions to the ground state, which also has singlet symmetry. Singlet emission was the basis for the earliest OLEDs. Triplets, on the other hand, have even symmetry and hence are quantum mechanically forbidden to transition to the singlet ground state. However, the selection rule that prevents their relaxation can be perturbed, leading to slow and very inefficient phosphorescence - a process that is not interesting for display applications. Unfortunately, simple statistical arguments show that an injected electron into an organic medium will excite singlet states only $25 \%$ of the time, with the other $75 \%$ of the electrons wasted on triplet state formation. This constraint is eliminated by the introduction of a heavy metal atom such as Pt or Ir into the luminescent molecule. The atom has a large orbital angular momentum, which when mixed with the electron spin in the organic ligand, results in spin-orbit coupling that leads to violation of the spin selection rule. This is the process of electrophosphorescence that can make every excited molecular state radiative, leading to $100 \%$ internal quantum efficiency. With this innovation, the electrophosphorescent OLEDs (PHOLEDs) became the most efficient light emitters known. 
Display manufacturers, notably Samsung in the Republic of Korea, took immediate notice of benefits of electrophosphorescence, and the OLED display revolution was off and running. In rapid succession, the Galaxy smartphone series featuring efficient, emissive and surprisingly long lifetime OLED displays was introduced, followed by LG Display producing large, ultrathin and attractive OLED televisions. And now, Apple iPhones and iWatches with OLED displays are flooding the marketplace, bringing OLEDs to a dominant position for information displays supporting a $\$ 25$ billion panel industry.

The success of OLEDs in the display industry naturally leads us to the question: what is next? Is there another "OLED revolution" waiting just around the corner for organic electronic materials and systems? Before we can answer that question, we must first consider what special attributes are offered by organic semiconductors that are not easily accessed by incumbent and proven inorganic semiconductors. After all, trying to displace an already served and mature market with an upstart technology is generally a fruitless exercise of catch-up, doomed to failure from the start. Listed below are several defining characteristics that point toward application spaces that might best be filled by organic electronics:

1. Optoelectronics: The very high absorption coefficients and often $100 \%$ emission efficiency of organic semiconductors make them an ideal platform for optoelectronic device applications (e.g., for light emitting displays and illumination, photodiodes and solar cells, etc.). Their electronic properties alone, as exploited in thin film transistors, do not generally provide organic electronics a clear "competitive edge" over conventional thin film semiconductor technologies. But when combined with ultrathin substrates, there are also some
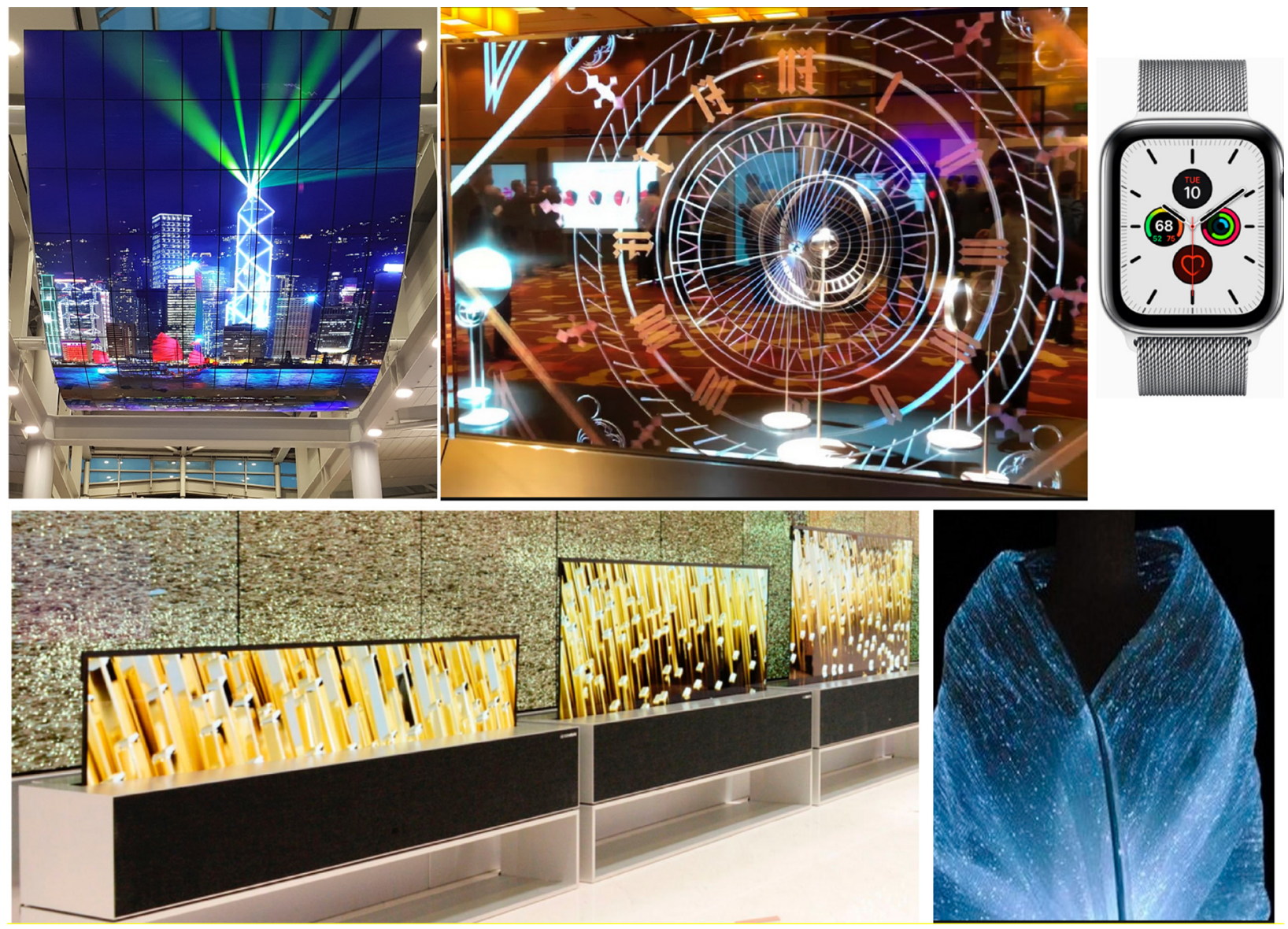

The many faces of organic light-emitting diode (OLED) displays. Beyond smart phones and OLED TVs, the flexibility and scalability of OLEDs has led to a plethora of new form factors and applications. Just a few examples are provided here. Clockwise from top left: Enormous OLED display on exhibit at the ceiling of Seoul-Incheon International Airport. Transparent OLED TV as part of a museum exhibit. Apple iWatch whose volume exceeded that of all Swiss watches sold in 2019. Concept of an OLED illuminated shawl. Rollable OLED (called ROLEDs by LG Display) TVs being deployed to varying heights. 
intriguing possibilities for purely electronic technology, as discussed below.

2. Materials diversity: The variety of organic molecules that can be developed is limitless. Hence, virtually every application need can be satisfied by engineering molecules that are optimized for a desired function.

3. Large area: Given the low cost and abundance of carbon-based materials comprising organic semiconductors, and their ability to be deposited onto substrates of almost arbitrarily large scale, they are ideally suited to applications where large area is a benefit. Displays provide an excellent example. Today, OLED televisions up to $77^{\prime \prime}$ diagonal are on the market. Lighting and solar cells are other examples that benefit from large area.

4. Flexibility, conformability, foldability: An unusual feature of organic electronics is that the devices are very thin (typically only a couple of hundred nanometers), employ very flexible, van der Waals bonded thin films and can be deposited onto nearly any flat substrate at low temperature. Hence, organic electronics is easily supported by ultrathin glass, plastic and metal foils. Roll-up and foldable displays are already entering the marketplace owing to this feature. Flexibility also lends itself for their use in "wearable electronics" that can be molded to complex shapes needed for watches, garments and a myriad of other applications. Conformability is also a useful attribute for interior lighting, shaped instrument panels and lighting fixtures such as tail lights in automobiles. Even devices made to conform to the irregular surfaces of living organisms can provide a significant application space for organic electronics.

5. Environmental friendliness: Since large area devices are often ubiquitously deployed, it is essential that the technology be nontoxic and easily disposed. Thankfully, organic electronic devices rarely contain materials with significant negative environmental or health impacts. The low deposition and processing temperatures used in organic device fabrication (typically at or only slightly above room temperature) imply a low energy investment and hence a comparatively small environmental impact from their large-scale manufacture.

6. Low cost: Production on flexible substrates suggests that organic electronic appliances can be produced in a continuous, high speed, roll-to-roll web process. Organic electronic semiconductors are closely related to inks, paints and dyes used in volume production of newsprint, fabrics, food packaging and a multitude of other common consumer products. These ultrahigh volume production methods are ideally suited to generating the large area devices that are the primary domain of organic devices. Indeed, unless a technology is low cost, there is little reason to believe that it will fill a niche where large area is demanded.

The list of characteristics common to organic electronic devices is undoubtedly longer than the six noted above. Yet, there are few if any thin film electronic technologies that have this collection of attributes that can open the door to many possible applications that remain unaddressed by incumbent materials and systems. With this introduction, we will devote the rest of this paper to answering the question, "What is the next big breakthrough technology that will be served by organic semiconductors?" These materials have set the stage with a brilliant "Act 1: Organic light emitting displays". So what does Act 2 look like, when will it arrive on the stage, and will it hold our attention as well as the opening act?

\section{OLEDs for lighting}

The value of OLEDs lies in their high efficiency, brilliant colors, flexibility and long operational lifetimes. These are the characteristics needed for all modern lighting fixtures that are now replacing the incandescent light bulb that has been illuminating indoor spaces while wasting an unconscionable amount of energy for over a century. There are advantages and disadvantages to OLED lighting that must be understood before it can become a widespread commodity. Among its disadvantages is that the intensity of an OLED is low compared to conventional LEDs based on InGaN (see Table 1). Thus, to provide sufficient luminosity to light up a room, a large OLED fixture is required, and this increases cost. One common way to increase brightness is to stack individual OLED elements separated by transparent charge generation layers (CGLs), as shown in Figure 3. For example, an electron injected into the OLED element nearest to the cathode draws a hole from the adjacent CGL, forming an exciton that subsequently radiatively recombines. The loss of a hole creates charge imbalance in the CGL, compelling it to emit an electron into the second OLED in the stack. This, in turn, draws a hole from the next lower CGL, creating the second radiative exciton, and so on until the entire stack is once again restored to neutrality. Hence, a single injected charge generates as many photons as there are OLED subelements, resulting in a luminosity that is multiplied times the number of stacked OLEDs. This not only increases luminosity but also increases quantum efficiency well above $100 \%$ (but not the power efficiency which is 


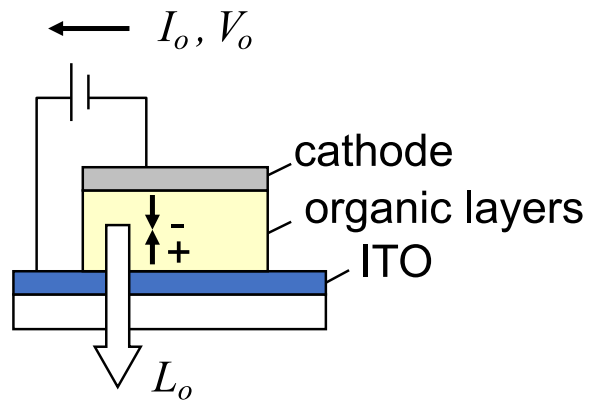

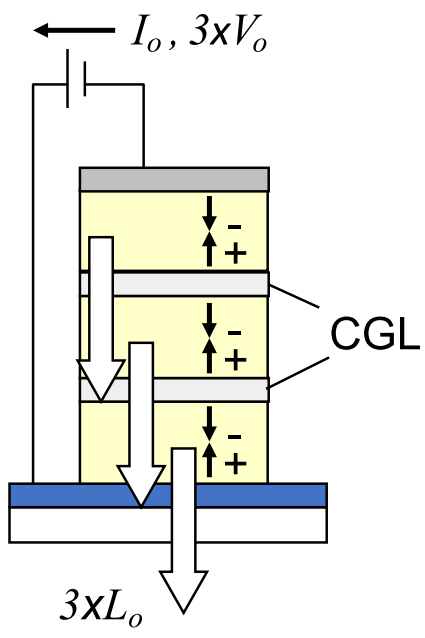

Figure 3: Comparison of a single element organic light-emitting diode (OLED) following thegeneric design offigure2andastackedOLED comprising three OLED subelements separated bytransparent charge generation layers (CGLs). The CGLs should be nearly optically and electronically lossless. In this case, the current $\left(I_{0}\right)$ and voltage $\left(V_{0}\right)$ required to produce the luminance, $L_{0}$, in the single element device are $I_{0}, 3 V_{0}$ to produce $3 L_{0}$ in the stacked device. Note that the lower current required to produce triple the luminositymakes the stacked structureideal for use in high intensity lighting applications. The CGL conventionally comprises a transparent oxide (e.g., $\mathrm{MoO}_{x}$ ) with thin, highly conductive electron and hole injecting films adjacent to the electron transport layer (ETL) and hole transport layer (HTL) of the contacting OLED subelements. constrained by the law of conservation of energy). To create white light, each subelement can emit in a different zone of the visible spectrum. Alternatively, the red, green and blue emitting molecules can be blended within each subelement (or a combination of subelements), each emitting in an appropriate proportion to provide illumination with the desired color temperature and color rendering index.

The ability to make large white emitting OLED fixtures can mitigate their relatively low brightness. In fact size combined with flexible substrates provides OLED lighting sources with their greatest advantage. An OLED does not require mounting in reflective, light-directing or distributing structures, known as luminaires. To date, all other lighting sources must be mounted in one of these costly fixtures. Yet, the flexible and conformable form factor of an OLED allows it to be shaped to form its own luminaire. For this reason, OLEDs provide architects with possibilities to custom design attractive lighting sources that would be very difficult to achieve using LEDs, fluorescent bulbs or other illuminants. Another attraction of OLEDs is color tunability. By placing separately addressed red, green and blue OLED stripes in a closely spaced, sideby-side arrangement, their white hue, or color temperature, can be tuned according to the time of day, mood or current purpose of the space being illuminated.

Table 1: Comparison of lighting sources.

\begin{tabular}{|c|c|c|c|c|}
\hline & Incandescent & Fluorescent & LED & OLED \\
\hline Efficacy & $17 \mathrm{~lm} / \mathrm{W}$ & $100 \mathrm{~lm} / \mathrm{W}$ & $\begin{array}{l}80-90 \mathrm{~lm} / \mathrm{W} \text { - white } \\
65 \mathrm{~lm} / \mathrm{W} \text { - warm white } \\
240 \mathrm{~lm} / \mathrm{W} \text { - lab demo }\end{array}$ & $\begin{array}{l}120 \mathrm{~lm} / \mathrm{W} \\
\text { Lab demos }\end{array}$ \\
\hline $\begin{array}{l}\text { Color rendering } \\
\text { index }\end{array}$ & 100 & $80-85$ & $\begin{array}{l}80 \text { - white } \\
90 \text { - warm white }\end{array}$ & Up to 95 \\
\hline Form factor & Heat generating & $\begin{array}{l}\text { Long or compact gas filled } \\
\text { glass tube }\end{array}$ & $\begin{array}{l}\text { Point source high intensity } \\
\text { lamp }\end{array}$ & $\begin{array}{l}\text { Large area thin diffuse } \\
\text { source } \\
\text { Flexible, transparent }\end{array}$ \\
\hline Safety concerns & Very hot & Contains mercury & Very hot in operation & None to date \\
\hline Lifetime (K h) & 1 & 20 & 50 & 40 \\
\hline Dimmable & $\begin{array}{l}\text { Yes, but much lower } \\
\text { efficacy }\end{array}$ & Yes, efficiency decreases & Yes, efficiency increases & Yes, efficiency increases \\
\hline Noise & No & Yes & No & No \\
\hline Switching lifetime & Poor & Poor & Excellent & Excellent \\
\hline Color tunable & No & No & Yes & Yes \\
\hline Cost & $\$ 0.50 / \mathrm{klm}$ & $\$ 1 / \mathrm{klm}$ & $\$ 3 / \mathrm{klm}$ & $\$ 50-100 / \mathrm{klm}$ \\
\hline
\end{tabular}


A comparison of performance of OLEDs and other lighting sources is provided in Table 1. With the exception of their high cost, OLEDs fill a niche for highly efficient, pleasant, indirect (soft) interior lighting. But cost is certain to come down in the next few years, primarily driven by the momentum and experience gained in the massive production of large and small screen displays. Ultimately, the cost of OLED lighting will be determined by the cost of encapsulations and substrates that must be impermeable to water and oxygen to prevent degradation of the organic materials. Also, for large area devices, the cost of the organic materials, although low compared to conventional inorganic semiconductors, becomes a factor. Nevertheless, the unique attributes of OLED lighting and the maturity of OLED technology suggest that lighting is poised to become a significant market for organic electronic devices.

\section{Organic solar cells}

Solar cells would appear to fit all the criteria to which organics are suited. They have large area, require the use of nontoxic materials, they take a relatively small energy investment in their large-scale manufacture and they can conform to whatever surface to which they are attached. The reason they are not widely deployed today is that their efficiency and lifetime, until recently, have been inadequate. Indeed, the increase in efficiency has been slow to materialize. After the $1 \%$ efficiency of a bilayer organic photovoltaic (OPV) was demonstrated, no major increase or development emerged until 10 years later with the introduction of the bulk heterojunction (BHJ) solar cell active region $[8,9]$. The $\mathrm{BHJ}$ is an entangled complex of donor and acceptor materials that eliminate the competition between the relatively long optical absorption length in organics $(\sim 100 \mathrm{~nm})$ compared to the diffusion length of excited states $(\sim 10 \mathrm{~nm})$ that must find their way to the donor-acceptor junction where they dissociate into a free electron and hole (see Box). Contemporaneous with the demonstration of the $\mathrm{BHJ}$ was the introduction of fullerene acceptors in both polymers [9] and small molecule [10] cells to replace the inefficient perylene diimide acceptors of the original bilayer cell. This led to a ten-fold increase in cell efficiency over the course of the next decade. Then, once again, materials innovations led to further efficiency increases by the introduction of thiophene-based "nonfullerene acceptors" [11]. Today, the efficiency of organic solar cells is approximately $17 \%$ and will soon reach $20 \%$ and beyond. In effect, advances in materials and structure have eliminated the complaint that "organic solar cells are not very efficient”.

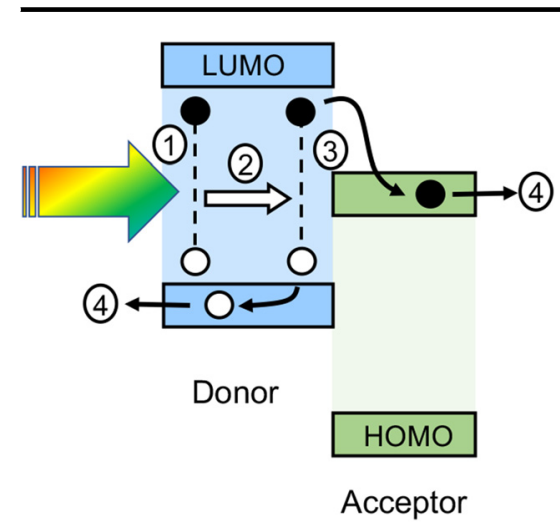

The photogeneration process in organics involves four steps. (1) Light incident (left) is absorbed in either the donor (D) or acceptor (A) layer (here shown only for $D$ absorption), creating an exciton. (2) The exciton diffuses to the D-A heterojunction $(\mathrm{HJ})(3)$ where it encounters an energy step. The electron transfers to the $A$, dissociating the exciton into a free electron and hole. (4) The electron drifts to the cathode and the hole to the anode. In organics, the conduction band is replaced by the lowest unoccupied molecular orbital level and the valence band by the highest occupied molecular orbital (the LUMO and HOMO, respectively). These are the "frontier orbitals" of the organic molecules themselves. This diagram illustrates a planar HJ. The electron and hole are indicated by filled and open circles, respectively, and the bound exciton state by the dashed lines. Most organic photovoltaics employ a bulk $H J$ where the $D$ and $A$ regions are intermixed at the nanometer scale to improve the probability that the exciton diffuses to the $\mathrm{HJ}$ without first recombining.

But what about reliability? A pervasive myth about organic materials and devices is that they lack the capacity for long-term reliability that we expect of our electronic appliances. Table 2 provides a compilation of lifetimes of OLEDs of the type used in displays, giving a hint to what makes some materials and structures more reliable than others. Generally, it is found that red pixels live longer than green pixels, and green live longer than blue. A reasonable conclusion, therefore, is that higher emission energies lead to shorter lifetimes. Indeed, this has been found to be the case. The primary source of molecular degradation is from high energy excitons colliding with other such excitons within the emission layer, and subsequently delivering sufficient energy to a molecular bond to break it [12]. Many strategies have been devised to limit the occurrence of such high energy excited state annihilation reactions, allowing for very long lifetimes of OLEDs now used in billions of displays.

In this same vein, fullerene-based OPVs have demonstrated remarkably long intrinsic lifetimes, extending over thousands of years [15]. But there is a difference between intrinsic lifetime and lifetime in the field where the packaged devices are exposed to the elements, from bright 
Table 2: Example lifetimes of OLEDs [2].

\begin{tabular}{|c|c|c|c|c|}
\hline OLED type & $\begin{array}{r}\text { Chromaticity } \\
\text { coordinates }\end{array}$ & $\begin{array}{r}\text { Luminous } \\
\text { efficiency } \\
(c d / A)\end{array}$ & $\operatorname{LT95}^{\mathrm{a}}$ (h) & LT50 (h) \\
\hline \multicolumn{5}{|l|}{ PHOLED ${ }^{\mathrm{b}}$} \\
\hline Deep red & $(0.69,0.31)$ & 17 & 14,000 & 250,000 \\
\hline ed & $(0.64,0.36)$ & 30 & 50,000 & 900,000 \\
\hline ellow & $(0.44,0.54)$ & 81 & 85,000 & $1,450,000$ \\
\hline Green & $(0.31,0.63)$ & 85 & 18,000 & 400,000 \\
\hline Light blue & $(0.18,0.42)$ & 50 & 700 & 20,000 \\
\hline \multicolumn{5}{|l|}{ Fluorescent $^{\mathrm{c}}$} \\
\hline Red & $(0.67,0.33)$ & 11 & & 160,000 \\
\hline Green & $(0.29,0.64)$ & 37 & & 200,000 \\
\hline Blue & $(0.14,0.12)$ & 9.9 & & 11,000 \\
\hline \multicolumn{5}{|l|}{$T A D F^{\mathrm{d}}$} \\
\hline Green $^{\mathrm{e}}$ & $(0.34,0.58)$ & 15 & $1380^{\mathrm{g}}$ & \\
\hline Light blue $^{f}$ & $(0.18,0.34)$ & & & $40^{\mathrm{h}}$ \\
\hline \multicolumn{5}{|c|}{ 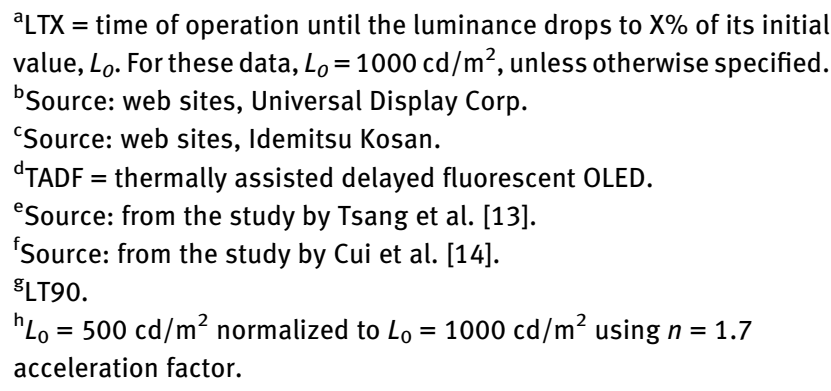 } \\
\hline
\end{tabular}

sunlight to rain, snow, ice, hail and dust. Furthermore, while the fullerene-based cells show this extraordinary endurance, the most efficient nonfullerene acceptor cells are much less robust, exhibiting lifetimes of only a couple of years [16]. Undoubtedly there is much work yet to be done to improve the lifetimes of the most efficient cells. Given the vast palette of materials available and that have yet to be synthesized and motivated by the unusual applications addressed by organic thin films, there is little doubt that both high efficiency and long device lifetimes will form the basis of a viable organic solar cell industry in the near future.

It is important to understand the appropriate niche for OPVs that distinguish them from incumbent solar technologies. The widespread generation of commodity power does not provide sufficient motivation for their development given the low costs enjoyed by Si panels. So, what can OPVs offer that Si solar cells cannot? The answer lies in the narrow but intense absorption spectra of organic molecules, allowing for very efficient solar cells that strongly absorb in the near infrared while being semitransparent, and importantly, neutral optical density in the visible. Such a solar module, deposited on a roll of plastic film, can be inserted in the pocket between the two sheets of glass

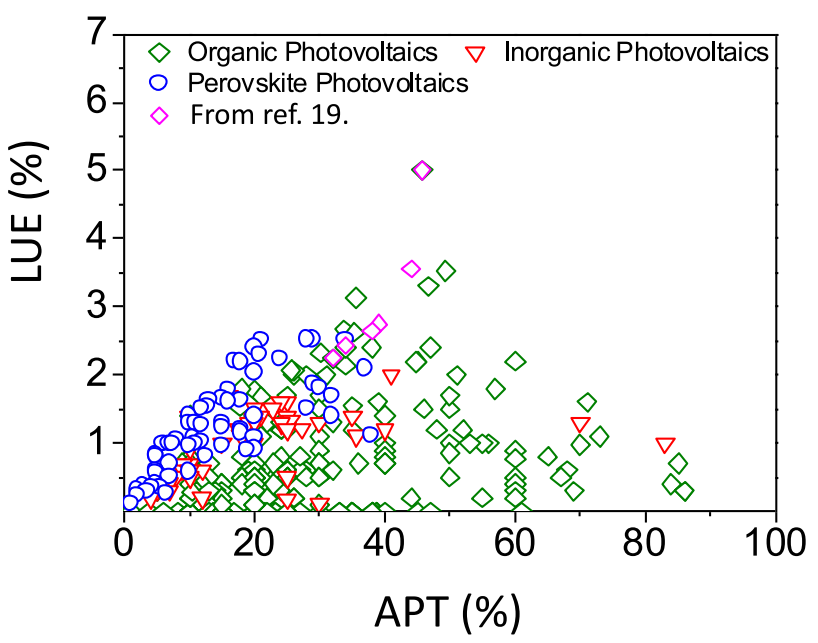

Figure 4: Compilation of selected results for the light utilization efficiency (LUE) versus the power conversion efficiency and the average photopic transparency (APT) for several different solar cell technologies. Here, LUE $=$ PCE $\times$ APT. Semitransparent organic photovoltaics (ST-OPVs) are noted by diamond symbols. Data obtained from the studies by Li et al. [17] and Traverse et al. [18]. PCE, power conversion efficiency.

forming a double pane window. Power generating windows, combined with microinverters, can supply considerable energy to buildings if they cover a reasonable fraction of a building surface. Such windows require an appropriate suite of materials to simultaneously provide high efficiency and visible transparency, along with optical coatings that reflect unabsorbed near infrared (NIR) radiation back into the cell for a second pass, while maximizing outcoupling of visible light. A figure of merit that quantifies the performance of semitransparent cells is their light utilization efficiency (LUE), which is the product of the PCE and the average photopic transparency (APT) of the cell. Here, the APT is the perceived transparency that is a convolution of the solar spectrum with the spectral sensitivity of the eye. Figure 4 shows a compilation of LUE vs. APT for thin film solar cells based on a range of technologies. There is little doubt that OPVs have a far more advantageous combination of these parameters than any other technology, including amorphous Si and perovskite solar cells.

An example of a high efficiency, neutral optical density organic solar cell is shown in Figure 5. It combines several solution-processed organic layers, semitransparent anode and cathode contacts, a visible optical outcoupling layer and an antireflection coating. This particular design has an 8.1\% PCE with APT $=43.3 \%$, leading to LUE $=3.5 \%$ [19]. The calculated thermodynamic efficiency limit for such single junction cells should eventually lead to 
(a)

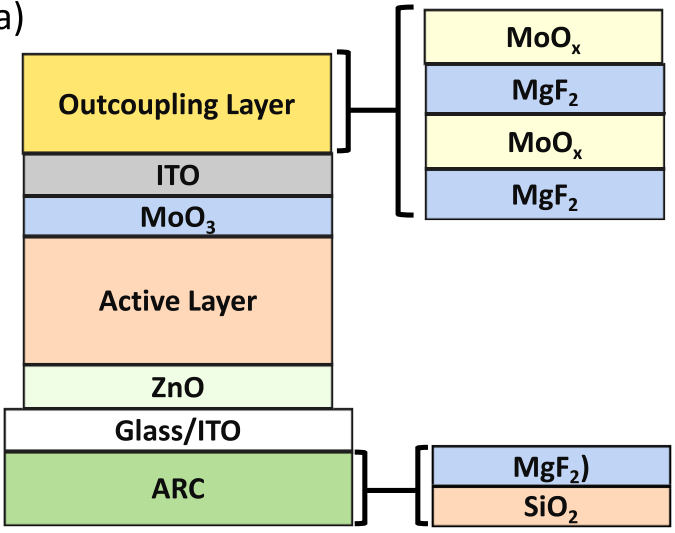

(b)

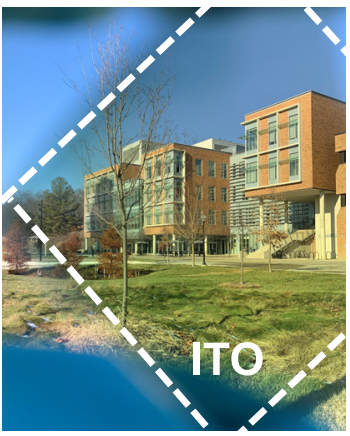

Figure 5: (a) Archetype structure of a high performance semitransparent organic photovoltaic (OPV) cell. The device consists of a glass substrate coated on its distal surface with a bilayer antireflection coating (ARC). The opposite surface comprises an ITO contact, a $\mathrm{ZnO}$ nanoparticle buffer layer. The bulk heterojunction active layer consists of a mixture of a solutiondeposited nonfullerene acceptor and a polymer donor. This is capped by a $\mathrm{MoO}_{3}$ electron conducting buffer layer and a second, transparent indium tin oxide (ITO) contact. The device is completed by the deposition of a four-layer outcoupling layer

that has a high transmission in the visible while it reflects the near infrared radiation back into the active layer for a second pass at absorption. (b) The solar cell appears nearly transparent with neutral density. In this case, the optical loss in the visible is approximately $50 \%$ [19].

LUE $>7 \%$, with even higher efficiencies achieved using multijunction versions.

As in other organic electronic devices, advances will be paced by innovations in materials. Yet the value proposition of ubiquitous solar generation on windows and other building surfaces is substantial. In this era where the production of carbon-free energy is no longer optional on our warming planet, coupled with the very low potential cost of organic devices rapidly produced in a roll-to-roll manufacturing process, it is nearly inevitable that OPVs will form the basis of Act 2 in the historical development of organic electronics.

\section{Organic transistors and beyond}

Beyond lighting and solar cells, the future opportunities in organic electronics become less clear. Recent advances in organic transistors, however, appear to open up possibilities for some electronic applications that, again, take advantage of the highly flexible form factors of organic thin film devices. The most common organic thin film transistor (OTFT) structure is a lateral geometry, with the organic channel deposited onto the surface of the gate insulator (see Box). The transistor operates in the accumulation mode: charge is drawn in to an otherwise undoped, large energy gap organic semiconductor channel from the source by the gate and drain potentials. As in conventional transistors, the channel current is modulated by the gate potential of $\sim 1 \mathrm{~V}$ in optimized devices. The field effect, or channel charge mobilities of organics $\left(<1 \mathrm{~cm}^{2} / \mathrm{Vs}\right)$ are less than or comparable to those of amorphous $\mathrm{Si}$ or metal oxide transistors, so mobility alone does not offer a competitive advantage in either transistor gain or bandwidth. In fact, while OTFTs have been the focus of research since their first demonstrations in the mid-1980s [20, 21], it has sometimes been asked if this is a technical solution looking for a problem.

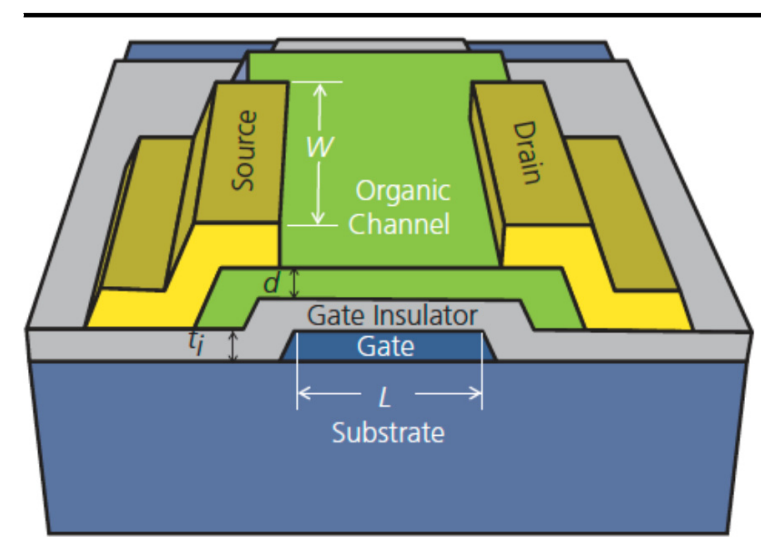

Common configuration of an organic thin film transistor. The source and drain are on the surface of the organic semiconductor channel, and the gate lies underneath the gate insulator. This is known as a bottom gate/top contact organic thin film transistor. Other geometries that switch the layering order of the insulator and gate contact, as well as the source and drain contacts, are also commonly employed.

But there are some interesting application niches for OTFTs that are not easily served by other electronic technologies. One is for selective detection of chemical compounds, agents or threats. The channels can employ organics that can bond or otherwise be altered in the presence of trace (parts per million or billion) concentrations of target chemicals (analytes). This, in turn, can result in a change in the interface charge density, thus shifting 
(a) Input biosignal from the heart

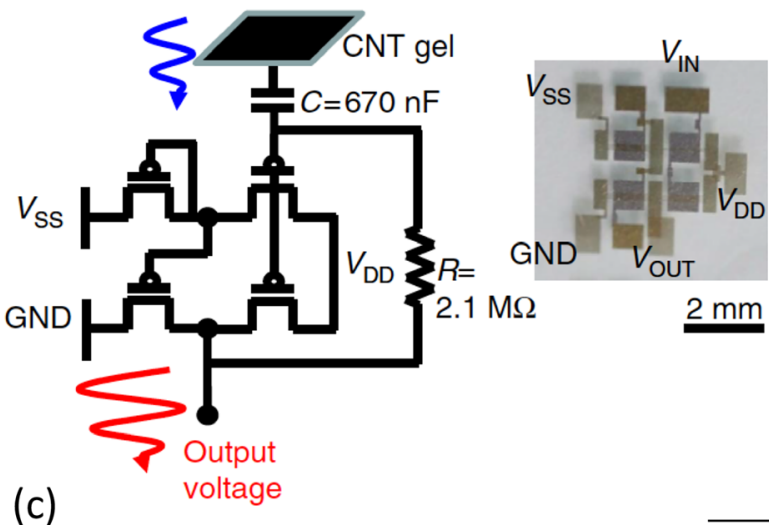

(b)

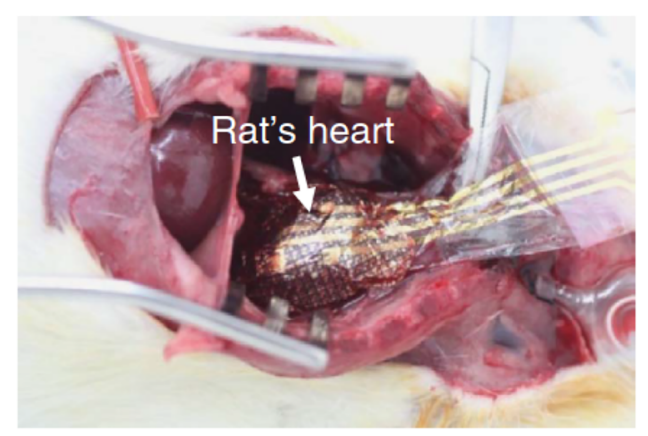

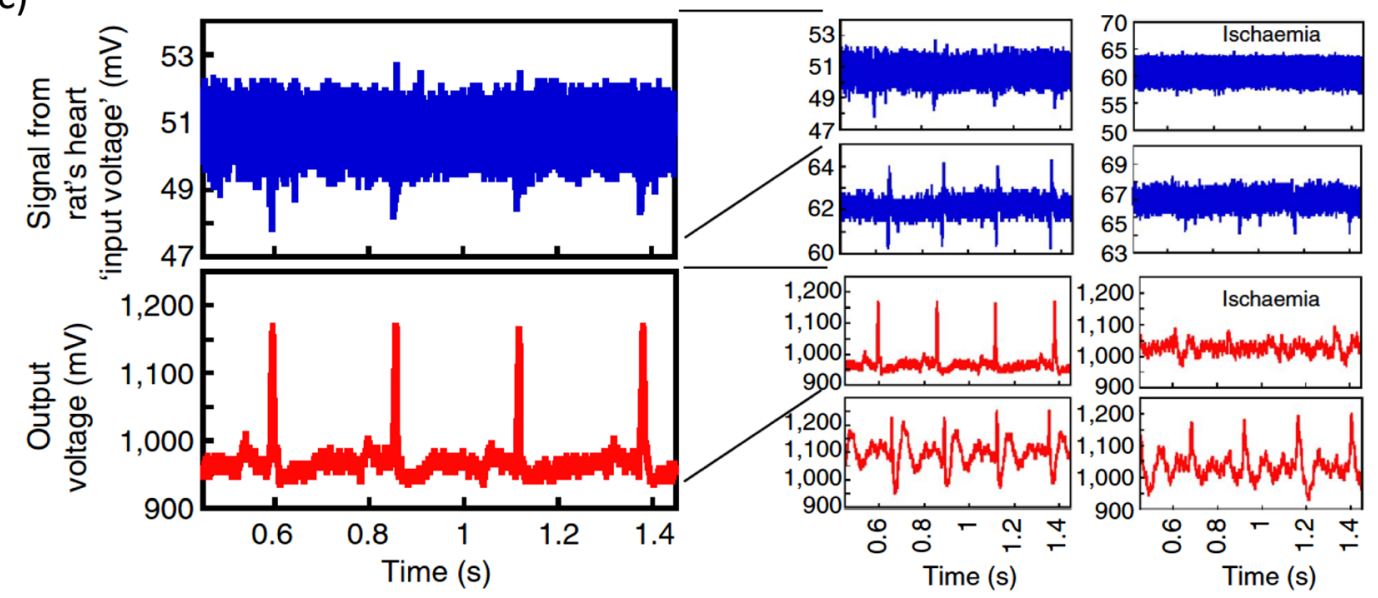

Figure 6: An example of imperceptible organic electronics used to monitor heart function in a rat.

(a) Amplifier circuit used in a biosensing array. Each pixel comprises a carbon nanotube (CNT) gel contact, input capacitor and amplifier circuit. A microscope image of the pixel is shown at right. (b) Photograph of the electrocaridio transducer attached to a rat's heart. (c) Electrocardiograms of the unamplified (blue) and amplified (red) heart impulses for several input conditions, including the ischemic state under myocardial infarction [23].

the transistor threshold voltage [2]. The response can be fast, highly selective, sensitive and reversible, making OTFT chemical sensors an interesting, large and diverse application opportunity.

An even more compelling application for OTFTs is in medical diagnostics. The transistors have been fabricated on plastic substrates that are only $\sim 1 \mu \mathrm{m}$ thick. Hence, the transistors can easily conform and adhere to irregular living tissues without impacting function. For this reason, organic electronic devices deposited on such ultrathin substrates have been termed "imperceptible electronics", which is yet another form of a wearable display [22]. An elegant example of such a device is the detector/amplifier array in Figure 6 used to monitor heart rhythm by placing the ultrathin electronic circuit in direct contact with the organ [23]. And while the niche for this particular device may be small, medical sensing diagnostics offers an almost limitless opportunity for organic electronics once an initial foothold is established. However, given the relative immaturity of organic electronics for chemical sensing, medical analyses, photodetection and so on, it will be some time before we are likely to see significant penetration by these technologies in the highly diversified but enormous application space of optoelectronic sensors that can be uniquely served by the attributes common to organic devices.

\section{Conclusions}

In this brief article, I have endeavored to answer the question of what is next in the field of organic electronics that will build on and extend the enormous initial successes of OLED displays? Displays are indeed a hard act to follow. But OLED lighting seems poised to serve interior illumination needs that complement existing inorganic LED lighting sources. I would classify this as Act 1 , Scene 2, for organics. It is not easy to predict how large an industry OLED lighting will grow into, given their high cost and relatively low 
luminosity, but they offer the architect a range of options that existing high brightness, specular LED sources do not. The second big opportunity for organics is in solar cells applied to windows and building facades. It is unlikely that they will ever displace Si (nothing ever does), so OPVs must capture markets not well served or that remain completely unserved by Si. Power windows and building applied photovoltaics seem to be applications that are ideally suited for OPVs. And finally, sensors based on OTFTs are an emerging opportunity whose boundaries are not yet known. Are there opportunities that have not been considered here? Indeed, they are as varied as are organic materials themselves. Memories, thermoelectric generators, one- and twodimensional quantum electronic devices, lasers and a range of other possibilities may yet emerge given the extraordinary versatility and variety of organic semiconductors [2]. But we do not have sight lines to these more distant possibilities. What we do know is that for this technology to succeed, it must exploit its unique attributes of large area, low cost, flexibility/conformability and environmental compatibility for it to win at opportunities not well served by conventional semiconductors. But when it comes to organic electronics, it is probably best to repeat a quote often attributed to the New York Yankee catcher, Yogi Berra: "It's tough to make predictions, especially about the future".

Author contribution: All the authors have accepted responsibility for the entire content of this submitted manuscript and approved submission.

Research funding: The author thanks the support from the US Office of Naval Research (contract no. N000142012114) US Air Force Office of Scientific Research (contract no. 17RT0908), the National Science Foundation (grant DMR 1905401), and Universal Display Corp.

Conflict of interest statement: The author declares an equity interest in Universal Display Corp. This apparent conflict of interest is under management by University of Michigan's Office of Research.

\section{References}

[1] H. Akamatu and H. Inokuchi, "On the electrical conductivity of violanthrone, iso-violanthrone, and pyranthrone," J. Chem. Phys., vol. 18, p. 810, 1950.

[2] S. R. Forrest, Organic Electronics: Foundations to Applications, Oxford, UK, Oxford University Press, 2020.

[3] C. W. Tang, "Two-layer organic photovoltaic cell," Appl. Phys. Lett., vol. 48, p. 183, 1986.

[4] C. W. Tang and S. A. VanSlyke, "Organic electroluminescent devices,” Appl. Phys. Lett., vol. 51, p. 913, 1987.
[5] M. A. Baldo, S. Lemansky, P. E. Burrows, M. E. Thompson, and S. R. Forrest, "Very high efficiency green organic light emitting devices based on electrophosphorescence," Appl. Phys. Lett., vol. 76, p. 4, 1999.

[6] M. A. Baldo, D. F. O’Brien, Y. You, A. Shoustikov, M. E. Thompson, and S. R. Forrest, "High efficiency phosphorescent emission from organic electroluminescent devices,” Nature, vol. 395, p. 151, 1998.

[7] C. Adachi, M. A. Baldo, M. E. Thompson, and S. R. Forrest, "Nearly $100 \%$ internal phosphorescence efficiency in an organic light emitting device," J. Appl. Phys., vol. 90, p. 5048, 2001.

[8] J. J. M. Halls, C. A. Walsh, N. C. Greenham, et al., "Efficient photodiodes from interpenetrating polymer networks," Nature, vol. 376, p. 498, 1995.

[9] G. Yu, J. Gao, J. Hummelen, F. Wudl, and A. J. Heeger, "Polymer photovoltaic cells - enhanced efficiencies via a network of internal donor-acceptor heterojunctions," Science, vol. 270, p. $1789,1995$.

[10] P. Peumans and S. R. Forrest, "Very high efficiency double heterostructure copper phthalocyanine/ $\mathrm{C}_{60}$ photovoltaic cells," Appl. Phys. Lett., vol. 79, p. 126, 2001.

[11] J. Hou, O. Inganäs, R. H. Friend, and F. Gao, "Organic solar cells based on non-fullerene acceptors,” Nat. Mater., vol. 17, no. 2, p. 119, 2018.

[12] N. C. Giebink, B. W. D’Andrade, M. S. Weaver, et al., “Intrinsic luminance loss in phosphorescent small-molecule organic light emitting devices due to bimolecular annihilation reactions," J. Appl. Phys., vol. 103, p. 044509, 2008.

[13] D. P.-K. Tsang, T. Matsushima, and C. Adachi, “Operational stability enhancement in organic light-emitting diodes with ultrathin Liq interlayers," Sci. Rep., vol. 6, p. 22463, 2016.

[14] L.-S. Cui, Y.-L. Deng, D. P.-K. Tsang, et al., "Controlling synergistic oxidation processes for efficient and stable blue thermally activated delayed fluorescence devices," Adv. Mater., vol. 28, no. 35, pp. 7620-7625, 2016.

[15] Q. Burlingame, X. Huang, X. Liu, C. Jeong, C. Coburn, and S. R. Forrest, "Intrinsically stable organic solar cells under high intensity illumination," Nature, vol. 573, p. 394, 2019.

[16] X. Du, T. Heumueller, W. Gruber, et al., "Efficient polymer solar cells based on non-fullerene acceptors with potential device lifetime approaching 10 years," Joule, vol. 3, no. 1, pp. 215-226, 2019.

[17] Y. Li, C. Ji, Y. Qu, et al., "High efficiency semi-transparent organic photovoltaics,” Adv. Mater., p. 1903173, 2019.

[18] C. J. Traverse, R. Pandey, M. C. Barr, and R. R. Lunt, “Emergence of highly transparent photovoltaics for distributed applications," Nat. Energy, vol. 2, no. 11, pp. 849-860, 2017.

[19] Y. Li, X. Guo, Z. Peng, et al., "Color-neutral, semitransparent organic photovoltaics," Proc. Natl. Acad. Sci. U. S. A., 2020, https://www.pnas.org/cgi/doi/10.1073/pnas.2007799117.

[20] G. Horowitz, D. Fichou, X. Peng, Z. Xu, and F. Garnier, “A fieldeffect transistor based on conjugated alpha-sexithienyl," Solid State Commun., vol. 72, no. 4, pp. 381-384, 1989.

[21] A. Tsumura, H. Koezuka, and T. Ando, "Polythiophene field-effect transistor: its characteristics and operation mechanism," Synth. Met., vol. 25, no. 1, pp. 11-23, 1988.

[22] T. Someya, S. Bauer, and M. Kaltenbrunner, "Imperceptible organic electronics,” MRS Bull., vol. 42, p. 124, 2017.

[23] T. Sekitani, T. Yokota, K. Kuribara, et al., "Ultraflexible organic amplifier with biocompatible gel electrodes," Nat. Commun., vol. 7, p. 11425, 2016. 